\title{
COMPUTER SIMULATION OF THE EVOLUTION OF AGN: SINGLE SUPERMASSIVE STAR VS. A CLUSTER OF MASSIVE WR STARS
}

\author{
R. SPURZEM, K. HERZIG \\ 1 Institut für Theoretische Astrophysik, Olshausenstr. 40, \\ D-2300 Kiel, F.R.G. \\ ${ }^{2}$ Universitäts-Sternwarte, Geismarlandstr. 11, \\ D-3400 Göttingen, F.R.G.
}

\begin{abstract}
The evolution of gas and stars in galactic nuclei is reconsidered and numerical models of early evolutionary phases of galactic nuclei are discussed, in particular the evolution of a supermassive gaseous object as a hypothetical precursor of a supermassive black hole.
\end{abstract}

\section{Introduction}

Since the discovery of quasars and of other types of active galactic nuclei (AGN) various theoretical models have been proposed to explain the characteristics of these exceptional astronomical objects. Among their extreme properties are the high luminosities $\left(\approx 10^{47} \mathrm{erg} \mathrm{s}^{-1}\right)$ produced on very small scales $(\approx 1 \mathrm{pc})$, the non-thermal emission spectra, polarization, the variability on short time scales, and the occurrence of jets. As the primary energy source the liberation of gravitational binding energy by the accretion of matter onto a supermassive central black hole (SMS) having a mass in the range of $10^{7} \mathrm{M}_{\odot}$ to $10^{9} \mathrm{M}_{\odot}$ has been suggested (Lynden-Bell, 1969) and is now commonly accepted (cf. e.g. Rees, 1984).

Tidal disruption of stars and accretion of the debris in the vicinity of the black hole cannot achieve such large masses within a Hubble time from a small seed black hole (Marchant and Shapiro 1980). Clues to the history of the supermassive black hole are processes like collisional disruption and/or coalescence of the stellar population of the nucleus and newly formed stars consisting of material liberated in stellar collisions or accumulated in the galactic centre from stellar mass loss in the outer regions.

Two basic scenarios are distinguished; first the Spitzer-Saslaw-Stone picture (Spitzer and Saslaw 1966; Spitzer and Stone 1967; see also Begelman and Rees, 1978; Illarionov and Romanova 1988): gas is liberated from disruptive stellar collisions if the central core velocity dispersion exceeds the escape velocity of individual stars. The gas settles as a thin disk of radius $R_{d}=\epsilon^{1 / 2} R$, where $R$ is the initial star cluster's radius and $\epsilon$ its initial eccentricity. Predominantly small stars $\left(0.1-0.5 \mathrm{M}_{\odot}\right.$, Illarionov and Romanova 1988) form out of the disk. Elastic encounters with disk and core stars and the Ostriker-Peebles instability enhance the velocity dispersion of such a stellar subsystem which again will suffer from liberation of gas and star formation. If the total luminosity of the resultant star-gas mixture reaches the Eddington limit star formation should cease and a supermassive star (SMS) containing a population of low-mass stars begins its subsequent evolution. We model the further development of such configuration by a computer simulation; the results are discussed below.

The other basic scenario is due to Colgate (1967) and Sanders (1970). They argue that coalescence of stars in the central region will lead to an agglomeration of several thousand massive stars $\left(M>100 \mathrm{M}_{\odot}\right)$; generally they are believed to produce an enhanced super-

K. A. van der Hucht and B. Hidayat (eds.),

Wolf-Rayet Stars and Interrelations with Other Massive Stars in Galaxies, 657-658.

(C) 1991 IAU. Printed in the Netherlands. 
nova rate and a cluster of compact stellar evolution remnants which ultimately collapses relativistically to a supermassive black hole (Zeldovich and Podurets, 1965; Shapiro and Teukolsky 1985). However, if coalescence timescales are short enough to proceed to a coherent supermassive object, one again ends, as in the Spitzer-Saslaw-Stone scenario, at the formation of a dense gas star system.

A more detailed numerical study of this evolutionary phase by means of a multicomponent model taking into account a mass spectrum of stars, coalescence and the dynamics of the liberated gas is subject of present work. For cloud systems in galaxies such a model was already utilized (Yorke et al. 1989).

\section{Conclusions}

Supermassive stars of more than about $5 \cdot 10^{5} \mathrm{M}_{\odot}$ are subject to the post-Newtonian dynamical instability during their $\mathrm{H}$-burning phase; they do not explode and thus form a supermassive black hole, provided their metallicity is below a critical value (Fuller et al., 1986). Usually such stellar evolution calculations deal with the evolution of an isolated supermassive star; Langbein et al. (1990) reported a first attempt to model numerically the evolution of a supermassive star containing a stellar system consisting of normal one solar mass single stars. Their calculations show that the energy generation by dissipation of stellar kinetic energy in the SMS exceeds the nuclear energy generation in certain evolutionary phases. Therefore we stress that the stellar evolution of such an SMS - provided it exists inside a galactic nucleus - may not be calculated without taking into account the stellar dynamical energy generation. The final fate of the SMS cannot yet be determined from the results of the simulations. The total amount of dissipated stellar kinetic energy alone, however, is smaller than the initial binding energy of the SMS. Future work in progress has to account for nuclear energy generation in combination with the stellar dynamical effects.

\section{Acknowledgements}

This work was supported in part by DFG grants Fr 325/23 and Yo 5/5; R. Sp. thanks the Institute of Astronomy and Astrophysics Würzburg and the Universitätssternwarte Göttingen for their kind hospitality.

\section{References}

Begelman, M.C., Rees, M.: 1978, Monthly Notices Roy. Astron. Soc. 185, 847

Colgate, S.A.: 1967, Astrophys. J. 150, 163

Fuller, G.M., Woosley, S.E., Weaver, T.A.: 1986, Astrophys. J. 307, 675

Illarionov, A.F., Romanova, M.M.: 1988, Astrophys. J. 32, 148

Langbein, T., Spurzem, R., Fricke, K.J., Yorke, H.W. : 1990, Astron. Astrophys. 227, 333

Lynden-Bell, D.: 1969, Nature 223, 690

Marchant, A.B., Shapiro, S.L.: 1980, Astrophys. J. 239, 685

Rees, M.: 1984, Ann. Rev. Astron. Astrophys. 22, 471

Sanders, R.H.: 1970, Astrophys. J. 162, 791

Shapiro, S.L., Teukolsky, S.A.: 1985c, Astrophys. J. 292, L41

Spitzer, L., Saslaw, W.C.: 1966, Astrophys. J. 143, 400

Spitzer, L., Stone, M.C.: 1967, Astrophys. J. 147, 519

Yorke, H.W., Kunze, R., Spurzem, R.: 1989, in Structure and Dynamics of the Interstellar Medium, Proc. IAU Coll. No. 120, eds. G. Tenorio-Tagle, M. Moles, J. Melnick, Springer, Berlin, p. 186

Zel'dovich, Ya. B., Podurets, M.A.: 1965, Astron. Zh. , 42, 963 (engl. translation in Sov. Astron., 9, 742). 